\title{
Cataract extraction in retinitis Pigmentosa patients
}

\author{
Abstract \\ Cataract is a common condition in retinitis pigmentosa eyes. This review aims at exploring \\ the particulars of cataract extraction in retinitis pigmentosa eyes.
}

Keywords: cataract, extraction, phacoemulsification, retinitis, pigmentosa, phimosis, dislocation, macular, edema, field
Volume 7 Issue 6 - 2017

\author{
Emad Selim \\ Eye Surgery Consultant Ltd, Edinburgh, UK
}

Correspondence: Emad Selim, I0I Rose street South Lane, Edinburgh,UK, Email emad.selim@outlook.com

\section{Introduction}

Retinitis pigmentosa is an inherited degenerative disease affecting retinal pigment epithelium and photoreceptors. Without cystoid macular edema, which can develop later in the course of the disease, central vision is normally preserved. When macula starts to become affected this can lead to central visual loss. ${ }^{1}$ Normally patients present with night blindness and restricted peripheral visual field. ${ }^{2}$ Examination of the retina shows arteriolar attenuation, bony spicules and pigmentary changes affecting the mid retinal periphery. ${ }^{3}$ Peripheral visual field testing shows generalized restriction. Electroretinography show low voltage waves and slow progression over time.

Like any other eye, eyes with retinitis pigmentosa can develop cataract. When cataract develops, it does not necessarily need operating unless it is visually significant. Most those needing interferences are posterior subcapsular cataract and they would normally need surgery at a younger age than cataracts without retinitis pigmentosa. ${ }^{4}$ Structural abnormalities were observed at electron microscopically levels in Anterior lens epithelium which lead to water influx into the lens with subsequent clouding along clefts which lead to posterior subcapsular lens opacities. Posterior subcapsular cataract is the type most frequently seen in patients with retinitis pigmontosa. When the right patients are chosen for surgery, cataract surgery can yield significant improvement in visual acuity. Patients' expectations shouldn't be set too high. ${ }^{5}$ Patient should be aware before surgery that central vision is what is likely to improve. Peripheral and dim vision is not likely to improve postoperatively. In case of dense cataract extraction, the peripheral visual field is likely to improve as well possibly to the field prior to the development of the dense cataract. Also glare caused by dense cataract is likely to improve the quality of visual function.

While assessing a retinitis pigmentosa patient for cataract surgeon, surgeons should keep in mind that favorable predictions of postoperative results can be misleading. However, it would help is patient is properly assessed thoroughly to avoid any unfavorable surprises. Careful fundus examination including macular examination is essential in ruling out macular abnormalities. If macular examination is not possible because of the density of cataract, examination of the fellow eye would give an idea of how the macula of the eye to operate would be like. However, this is not guaranteed to provide accurate assessment. Optical coherence tomography can help as well as fluorescein fundus angiography in the assessing the macular condition. Other potential visual acuity predictors can be used such as laser interferometry. To set patients' expectation right for the procedure, a trial of mydriasis for a couple of week can give an idea of how vision would be like postoperatively. ${ }^{5}$

\section{Visual potential post cataract surgery}

It general it is hard to tell patients how much their vision is likely to improve postoperatively in figures like lines down the chart because retinitis pigmentosa is a rare disease and most studies done to detect visual outcome were done on small number of patients. In one study on 30 patients, $83 \%$ improved two lines down the Snellen charts. ${ }^{6}$ Patients with a poor preoperative visual acuity normally do not improve significantly postoperatively which mainly due to macular edema.

\section{Common postoperative complications}

Anterior capsular contracture; It is essential to expect anterior capsular contracture postoperatively in retinitis pigmentosa eyes. Therefore, small capsulorhexis should be avoided. Perhaps the mechanism behind it was the zonular insufficiency which is common in retinitis pigmentosa. Similar anterior capsular contracture happens in eyes with similar zonular in sufficiency such as in diabetes mellitus, uveitis, pseudoexfoliation syndrome, high myopia and elderly patients. This should be treated promptly by creating radial YAG laser capsulotomies. ${ }^{7}$ Femtosecond laser pseudophakic capsulotomy can be used with advantage over the standard YAG capsulotomy.

Posterior capsular opacification occurs postoperatively in almost every retinitis pigmentosa eye after cataract surgery. Incidence in most studies is $100 \%$ at 6 months postoperatively. The incidence of posterior capsular opacification varies according to the type of lens inserted. Smooth hydrophobic lenses tend to create less fibrosis. ${ }^{8}$ Dislocation of intraocular lens is a common complication postoperatively. Complete dislocation of the bag-IOL complex is common. Some intraocular lenses are more likely to be dislocated than others. Silicon lenses are more likely to be dislocated specially if early capsulotomy is required. ${ }^{9}$ Cystoid macular edema is also a common complication of cataract surgery in retinitis pigmentosa eyes. Corticosteroids should be recommended postoperatively to help protect from CME. ${ }^{1}$ 


\section{Conclusion}

Retinitis pigmentosa is a rare hereditary disease affecting the retina. Cataract develops in retinitis pigmentosa eyes. At some stage cataract would need to be removed. Certain precautions should be taken when operating for cataract in retinitis pigmentosa eyes. Among these precautions is the size of anterior capsulorhexis which should be large enough to avoid anterior capsular phimosis and not too large to allow intraocular lens dislocation. Care should be taken to avoid increasing already there cystoid macular edema which would limit the benefit from cataract extraction if it gets worse.

\section{Acknowledgments}

None.

\section{Conflicts of interest}

Author declares that there are no conflicts of interest.

\section{Funding}

None.

\section{References}

1. Sandberg MA, Brockhurst RJ, Gaudio AR, et al. The association between visual acuity and central retinal thickness in retinitis pigmentosa. Invest Ophthalmol Vis Sci. 2005;46(9):3349-3354.
2. Harton DT, Berson EI, Dryja TP. Retinitis pigmentosa. Lancet. 2006;368(9549):1795-1809.

3. Pruett RC. Retinitis pigmentosa: clinical observations and correlations. Trans Am Ophthalmol Soc. 1983;81:693-735.

4. Fishman GA, Anderson RJ, Lourenco P. Prevalence of posterior subcapsular lens opacities in patients with retinitis pigmentosa. $\mathrm{Br} J$ Ophthalmol. 1985;69(4):263-266.

5. Yoshida N, Ikeda Y, Murakami Y, et al. Factors affecting visual acuity after cataract surgery in patients with retinitis pigmentosa. Ophthalmology. 2005;122(5):903-908.

6. Bastek JV, Heckenlively JR, Straatsma BR. Cataract surgery inretinitis pigmentosa patients. Ophthalmology. 1982;89(8):880-884.

7. Nikpoor N, Stone DU. Rapid capsular phimosis in retinitis pigmentosa. Ophthalmic Surg Lasers Imaging. 2010;41.

8. Bruno M, Nebbioso M, Rigoni E, et al. Posterior capsule opacity in retinitis pigmentosa according to different biomaterials of intraocular lenses: Our clinical experience. Clin Ter. 2015;166(5):191-193.

9. Masket S, Bostanci Ceran B, Fram NR. Spontaneous dislocation of posterior chamber intraocular lenses (PC IOLs) in patients with retinitis pigmentosa - case series. Saudi J Ophthalmol. 2010;26(1):61-65. 